\title{
High contrast artifact reduction in Cone Beam Computed Tomography by using Geometric Techniques
}

\author{
Peter B. Noël ${ }^{a, b}$, Jinhui Xu ${ }^{b}$, Kenneth R. Hoffmann ${ }^{a, b}$, Jason J. Corso ${ }^{a}$, Sebastian Schafer $^{b}$ \\ and Alan M. Walczak ${ }^{b}$ \\ ${ }^{a}$ Department of Computer Science and Engineering, The State University of New York at \\ Buffalo, USA. \\ ${ }^{b}$ Department of Neurosurgery (Toshiba Stroke Research Center), The State University of New \\ York at Buffalo, USA.
}

\begin{abstract}
The use of cone beam computed tomography (CBCT) is growing in the clinical arena, due to its ability to provide 3-D information during interventions, its high diagnostic quality (sub-millimeter resolution), and its short scanning times (10 seconds). In many situations, the reconstructions suffer from artifacts from high contrast objects (due mainly to angular sampling by the projections or by beam hardening) which can reduce image quality. In this study, we propose a novel algorithm to reduce these artifacts. In our approach, these objects are identified and then removed in the sinogram space by using computational geometry techniques. In particular, the object is identified in a reconstruction from a few views. Then, the rays (projection lines) intersecting the high contrast objects are identified using the technique of topological walk in a dual space which effectively models the problem as a visibility problem and provides a solution in optimal time and space complexity. As a result, the corrections can be performed in real time, independent of the projection image size. Subsequently, a full reconstruction is performed by leaving out the high contrast objects in the reconstructions. Evaluations were performed using simulations and animal studies. The artifacts are significantly reduced when using our approach. This optimal time and space complexity and relative simple implementation makes our approach attractive for artifact reduction.
\end{abstract}

\section{INTRODUCTION}

In the clinical arena, reconstruction of cone beam computed tomography (CBCT) data can result in situations where the image quality is reduced because of artifacts. The most common artifacts, such as streaks, result from insufficient angular sampling and/or beam hardening. Angular sampling artifacts are due to the object having higher spatial angular frequencies than the angular sampling. Beam hardening artifacts are due to the changing xray beam quality(higher average energy, or hardening) as the x-ray beam passes through the material. Examples of situations where these problems occur in the clinic are dental imaging (e.g., implants), total prostheses (e.g., hip), surgical imaging (e.g., metal clips), and vascular imaging (e.g., contrast filled vasculature).

Several groups have worked on correcting or enhancing the projection data. Zhao et. al. proposed a waveletbased multiresolution analysis method for metal artifact reduction, in which information is removed from the corrupted projection data. ${ }^{1}$ Also machine-learning approaches have been used where a maximum posteriori algorithm for reducing metal streak artifacts was used. ${ }^{2}$ Bal et. al. presented a novel method for metal artifact reduction by painting missing information into the corrupted sinogram. ${ }^{3}$ Another group developed an algorithm that utilizes a tilted parallel beam geometry to estimate the projection error and generate an error estimation image, which is then subtracted from the initial reconstruction. ${ }^{4}$

Our approach is distinct from previous work. It models the problem as a visibility problem and develops a highly computationally efficient way to reduce the artifacts. Inparticular, the rays (projection lines) intersecting the high contrast object are computed in dual space in optimal running time by walking on an arrangement which

Further author information: (Send correspondence to Peter B. Noël)

Peter B. Noël: E-mail: pbnoel@buffalo.edu, Telephone: (716) 829-3594 
the shape of the high contrast objects. As a result, independent of the projection image size, the corrections can be done in real time.

The rest of the paper is organized as follows. In sections 2.1 and 2.2, we revisit the filtered backprojection method and computational geometry tools, and in Section 2.3, we present our algorithm. In section 3, we show how the algorithm proposed is evaluated, in section 4 the results are shown. Finally, in section 5, a discussion is given.

\section{METHOD}

\subsection{Cone-Beam Computed Tomography (CBCT)}

In this section, we are revisiting a reconstruction method for CBCT data as introduced by Feldkamp et al. ${ }^{5}$ Since we use a rotational angiographic system equipped with a flat panel detector, we only discuss the case of equally spaced planar detectors.

In Figure 1, the schematic drawing of the cone beam system with a planar detector is presented. During acquisition, the system follows a circular trajectory, with a radius of $D$ placed at the origin. The detectorplane is perpendicular to the central axis of the x-ray beam.

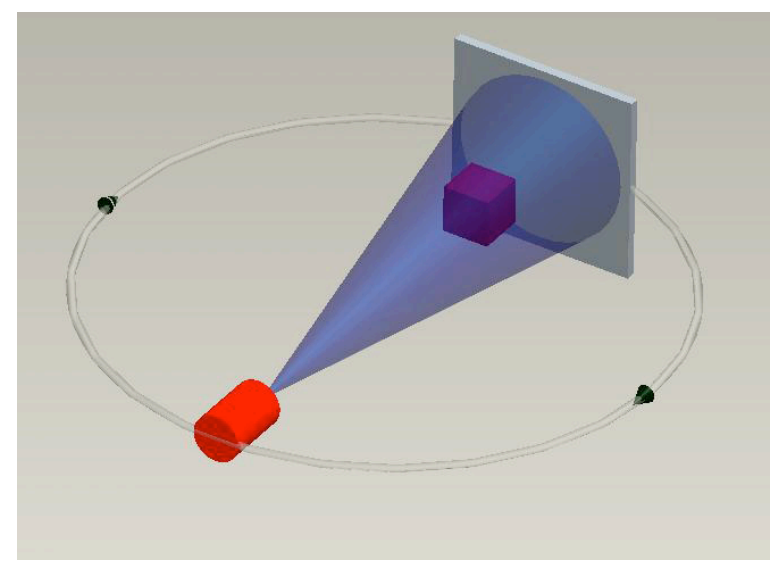

Figure 1. Systematic drawing of a cone beam system.

The projection image $P(\cdot)$ at angular position $\Theta$ is the line integral along the x-ray beam. A set of projections are acquired at $t$ discrete source positions with uniform angular spacing $\Delta \Theta$. During CBCT, $\Theta$ range is about $210^{\circ}$ with angular separations of $1^{\circ}$. A full rotation is not possible due to mechanical limitations.

The reconstruction method is formulated as a weighted filtered backprojection. As an initial step, the projection data are $\log$ converted, individually weighted, and ramp filtered $\left(P_{f}\right)$. Next, the 3-D volume is reconstructed by a backprojection. Let $r=[x, y, z]$ be the 3 -D position in the volume, and let $(u, v)$ denote the position of the intersection with the detector plane of the ray starting from the source and passing through point $r$. Therefore, the backprojection is:

$$
f(\vec{r})=\sum_{\Theta} P_{f}[u(x, y, \Theta), v(x, y, \Theta), \Theta],
$$

where

$$
\begin{aligned}
& u=(S I D * x) /(I S O-z), \\
& v=(S I D * y) /(I S O-z),
\end{aligned}
$$

SID is the source-to-image distance, and ISO is the isocenter distance. The isocenter is the point about which the system rotates and the isocenter distance is the source-to-isocenter-distance. Since $u$ and $v$ usually do not correspond to a discrete pixel position, we use interpolation to determine gray values in the image. The computational cost of cone-beam computed tomography for a volume of size $N^{3}$ is $O\left(N^{4}\right)$. 


\subsection{Object Identification}

As an initial step in our approach, a lower resolution reconstruction is performed using the filtered backprojection algorithm (see section 2.1). For this initial reconstruction, the number of projections used and the resolution depends upon the object size and the system parameters. Next, the selected high contrast objects are segmented. The segmentation can be done by user input or any automatic segmentation technique such as Active Shape Models. ${ }^{6}$ The segmented points along the edges are saved as vertices $\left\{v_{1}, v_{2}, \ldots, v_{m}\right\}$ of the polygon $V$, where each polygon corresponds to a high contrast object.

\subsection{Geometric Techniques for optimally rapid determination of projection rays intersecting the object}

With the object and the vertices identified, we then find the projection lines which intersect the object using point-line duality and visibility, as described below. To simplify the description, we discuss the 2D case, however the same rules and techniques can be applied to 3D data. Note, our implementation is done in 3D.

An arrangement $A(H)$, where $H$ is set of straight lines, is a decomposition of the embedding plane $P$ into a set of maximally connected convex regions induced by the $n$ lines in $H$ and their pairwise intersections. In general, $A(H)$ consists of $O\left(n^{2}\right)$ cells, edges, and vertices. All faces can be reported by sweeping and reporting them in an on-line fashion in $O\left(n^{2}\right)$ time and $O(n)$ space. To determine this information quickly, we use a theoretically optimal computational geometry technique called Topological Peeling ${ }^{7}$ or Topological Walk. ${ }^{8}$ Topological Walk / Peeling are algorithms for reporting line or ray intersections in a plane or volume. The main steps performed by topological walk: (i) construct a graph from the lines in dual space; (ii) compute the initial cut (Note, in graph theory, a cut $(C)$ is a partition of the vertices of a graph into two sets.); (iii) construct a upper tree $(T)$ (iv) starting at the root, use a dynamic depth-first search on the upper tree to find the left-most twig; (v) update $C$ and $T$ (vi) repeat steps (iv) and (v). In a certain way, Topological Peeling/Walk could be viewed as a "wave" propagating through the cells of the arrangement from one or more source points. The time and space complexities for these techniques are optimal.

Let $P$ denote the 2-D plane and consider the duality transform that maps a line $l: y=m x+p$ in $P$ onto the point $l^{*}:(m, p)$ on the dual plane $P^{*}$. Using this point-line duality, the set of lines passing through a point $q:(a, b)$ in $P$ corresponds to the line $q^{*}: y=-a x+b$ in $P^{*}$.
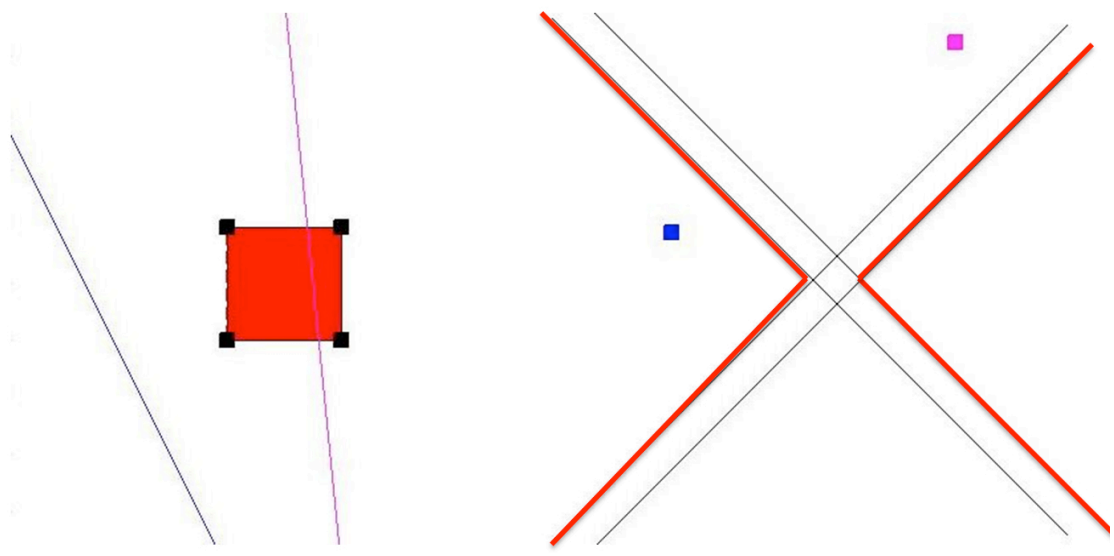

Figure 2. Primal (left) and dual (right) space generated by using point line duality. The object (spanned by black vertices) is hit by the magenta ray. The blue ray misses the object. In the dual space, the black vertices span the hourglass with black lines (the border of the hourglass is shown in red lines). The magenta point (the dual of the lighter ray) is within the hourglass, and the blue point is outside.

For all projections, the set of rays $L$, which start at the source point and end at each pixel position on the projection plane are loaded. A subset of these rays intersects with the convex polygon $V$, which represents the high contrast object. Using the point-line duality, we can transform each vertex of the polygon $V$ to a line $v$, and each ray $l$ to a point $l^{*}$ in dual space. In figure 2, an example for the primal and dual space is shown. An 
hour glass is the (unbounded) region between two disjoint unbounded chains, such that one chain is convex and the other one is concave. The union of straight lines in $\mathrm{V} *$ forms an hourglass in dual space and a simple convex polygon in its primal space. The subset of rays which intersect with $\mathrm{V}$ (the high contrast object) are located at points within the hourglass in dual space. Subsequently, the rays which do not intersect are placed as points outside the hourglass in dual space.

Theorem: The union of straight lines in $V^{*}$ forms an hourglass in dual space and a simple convex polygon in its primal space. The subset of rays which intersect with $V$ (the high contrast object) are located at points within the hourglass in dual space. Subsequently, the rays which do not intersect are placed as points outside the hourglass in dual space.

Proof: Follows from the definition of hourglass, duality transform and Lemmas in. ${ }^{7}$

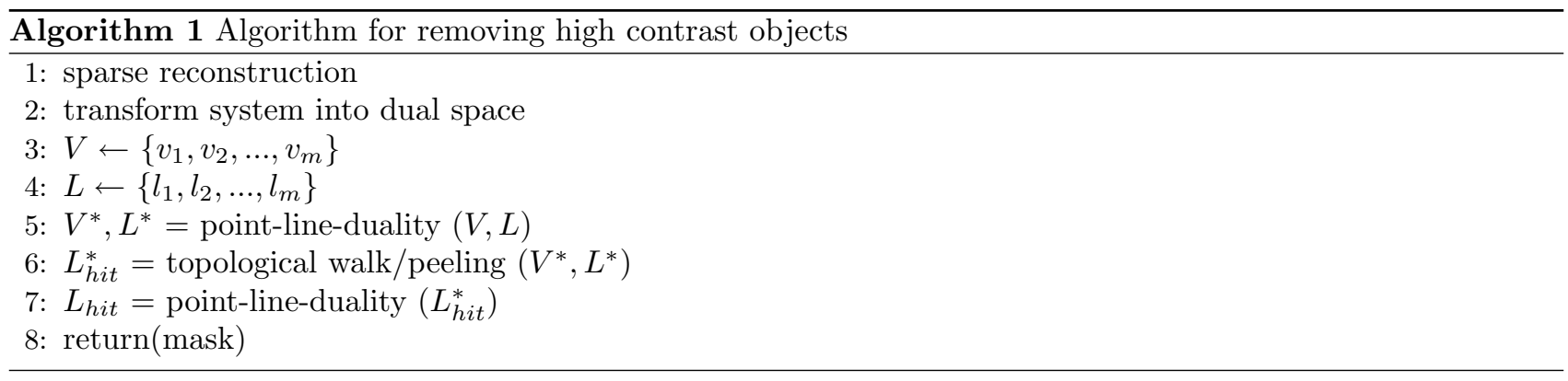

The arrangement of the dual lines and the point locations of $\mathrm{L}^{*}$ in the dual space are determined by using the topological walk/peeling algorithm. After this step, a mask is returned which describes the position of the high contrast objects as a function of the projection angle theta.

\subsection{Removing High Contrast Objects}

Next, the projection data are filtered. The process of filtering is minimally modified, i.e, the regions which are corresponding to the high contrast objects are left out during filtering. Finally, the full filtered reconstruction is performed, but the projection data associated with the earlier identified object data are not included. Therefore, the final reconstruction (see section 2.1) contains only the low contrast objects without the high contrast objects.

\section{EVALUATION}

We evaluated our algorithm using the Shepp-Logan phantom and real cone-beam computed tomography data.

\subsection{Shepp-Logan phantom}

For the simulations, the standard Shepp-Logan phantom was generated in Matlab. A high contrast object consisting of overlapping semi-circles was added to the initial phantom. Around the high contrast object, three smaller low contrast circles were placed (Figure 3). The phantom data then reprojected into 360 views (SID 123 $\mathrm{cm}$, ISO $82 \mathrm{~cm}$ ), with an angular separation of $1^{\circ}$ between projections.

\subsection{Animal Study}

In order to determine the performance of our algorithm on real data, we use data from a rabbit study. The CBCT system acquires 106 projections with an angular separation of $2^{\circ}$ and a matrix size of $1024 \times 1024$. The projections are not distortion corrected since our system is equipped with an flat panel detector. A metal sphere was attached to the skin of the rabbit. This high contrast object produced intense artifacts in the reconstruction. To reduce these artifacts, we used our new approach described above. 


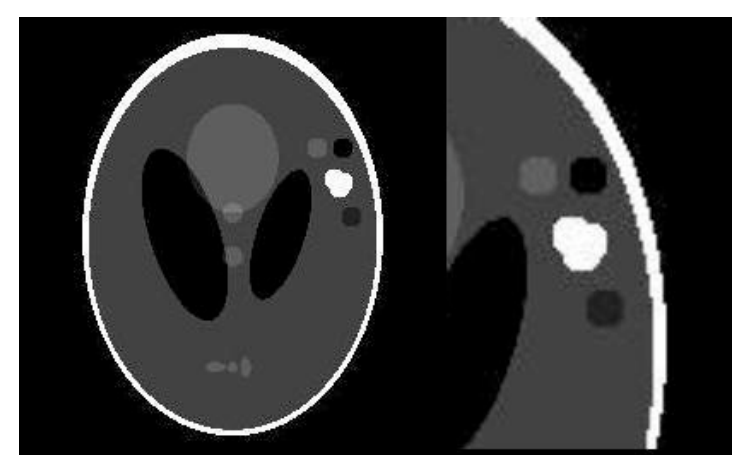

Figure 3. The modified Shepp-Logan phantom(left) and the modified region enlarged (right). Three low and one high contrast object have been added.

\section{RESULTS}

\subsection{Shepp-Logan phantom}

In Figure 4, we show the binary mask, returned after determining the intersecting rays, which shows the location of the high contrast objects as a function of projection angle. Additionally, we show the original projection data (sinogram) and the subtraction of the mask from the original projection data. The subtraction image shows that the results from our algorithm are accurate to within 1 pixel. Note, this error is always an overestimate.

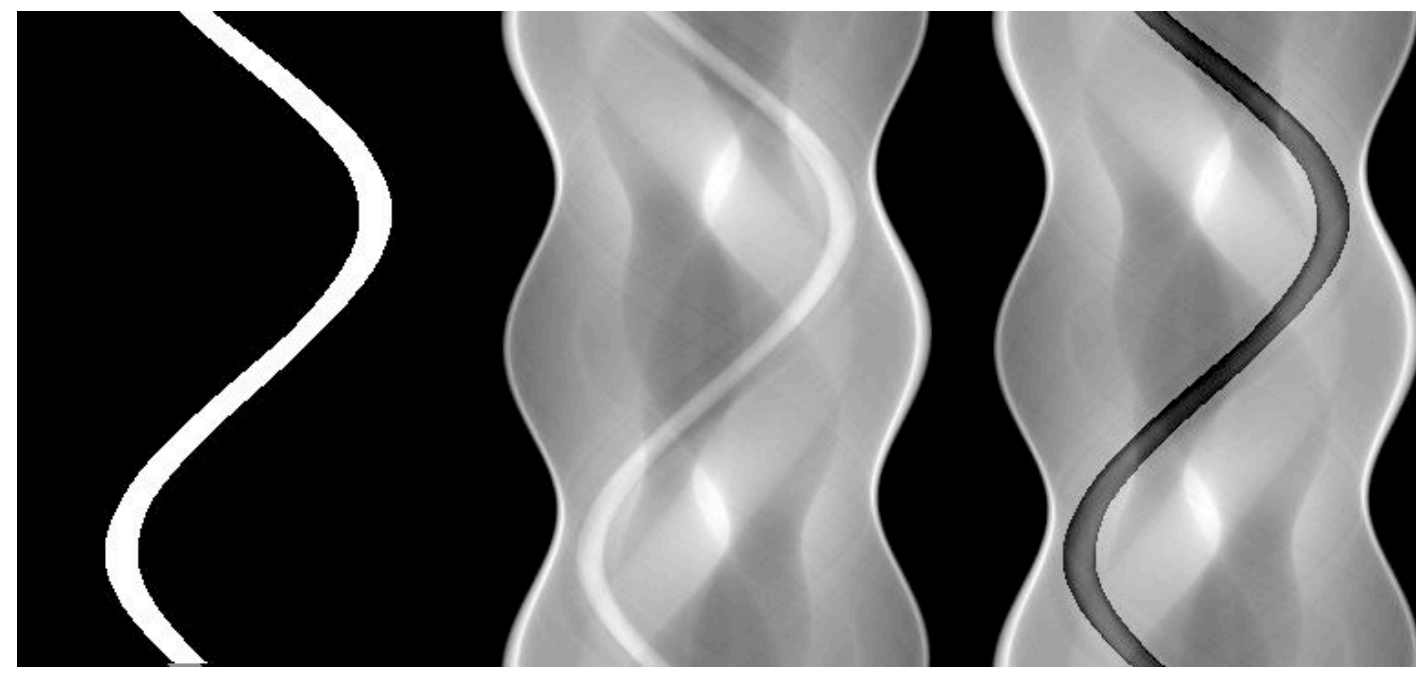

Figure 4. The returned mask (left), the original sinogram (middle), the subtraction of the previous two (right). Note the result from our algorithm is correct within one pixel.

The final result of the Shepp-Logan study is presented in Figure 5. On the left side, the reconstruction without using any correction is shown. On the right side, the result after using our algorithm shown. Note, the low contrast objects around the removed object are clear and visible. The image quality is improved due to reduced artifacts. The correction is done in real time.

\subsection{Animal Study}

The final result of the animal study is presented in Figure 6. On the left side, the reconstruction, obtained using standard reconstruction techniques is shown. On the right side, the result after using our algorithm is shown. Note, the low contrast objects are significantly more visible after enhancing the information with our approach. The image quality is improved due to reduced artifacts. The correction was done in real time (2 seconds). 


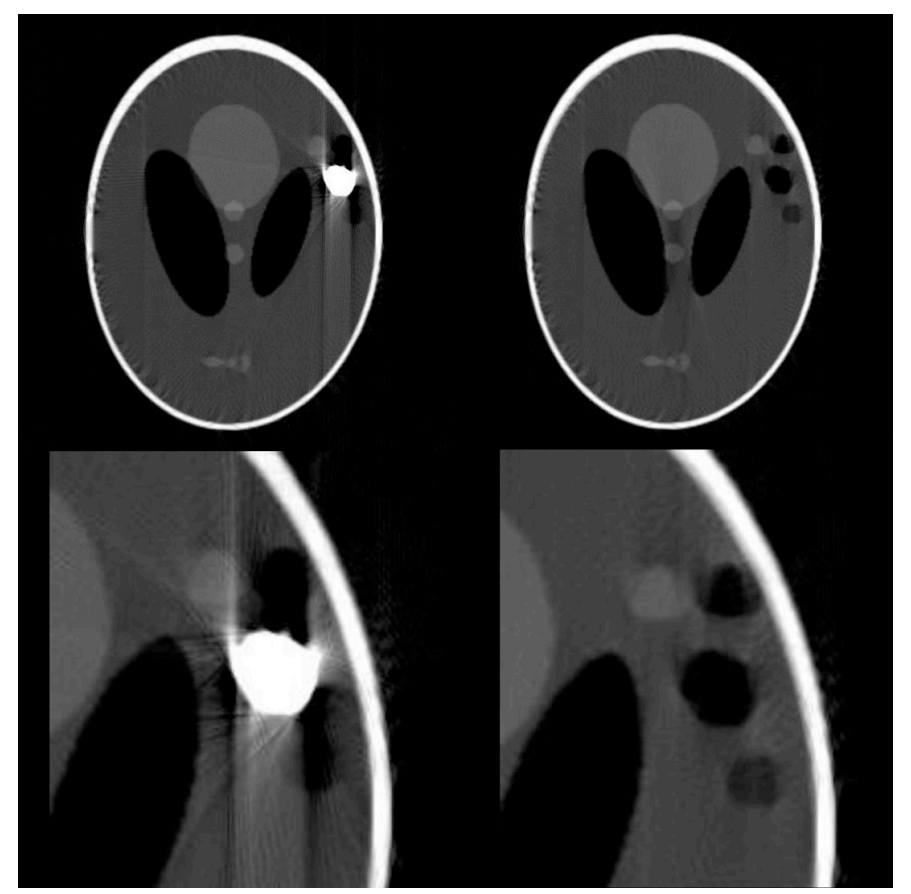

Figure 5. The resulting reconstruction without correction (left), and with correction (right). The lower row shows the enlarged regions of interest.

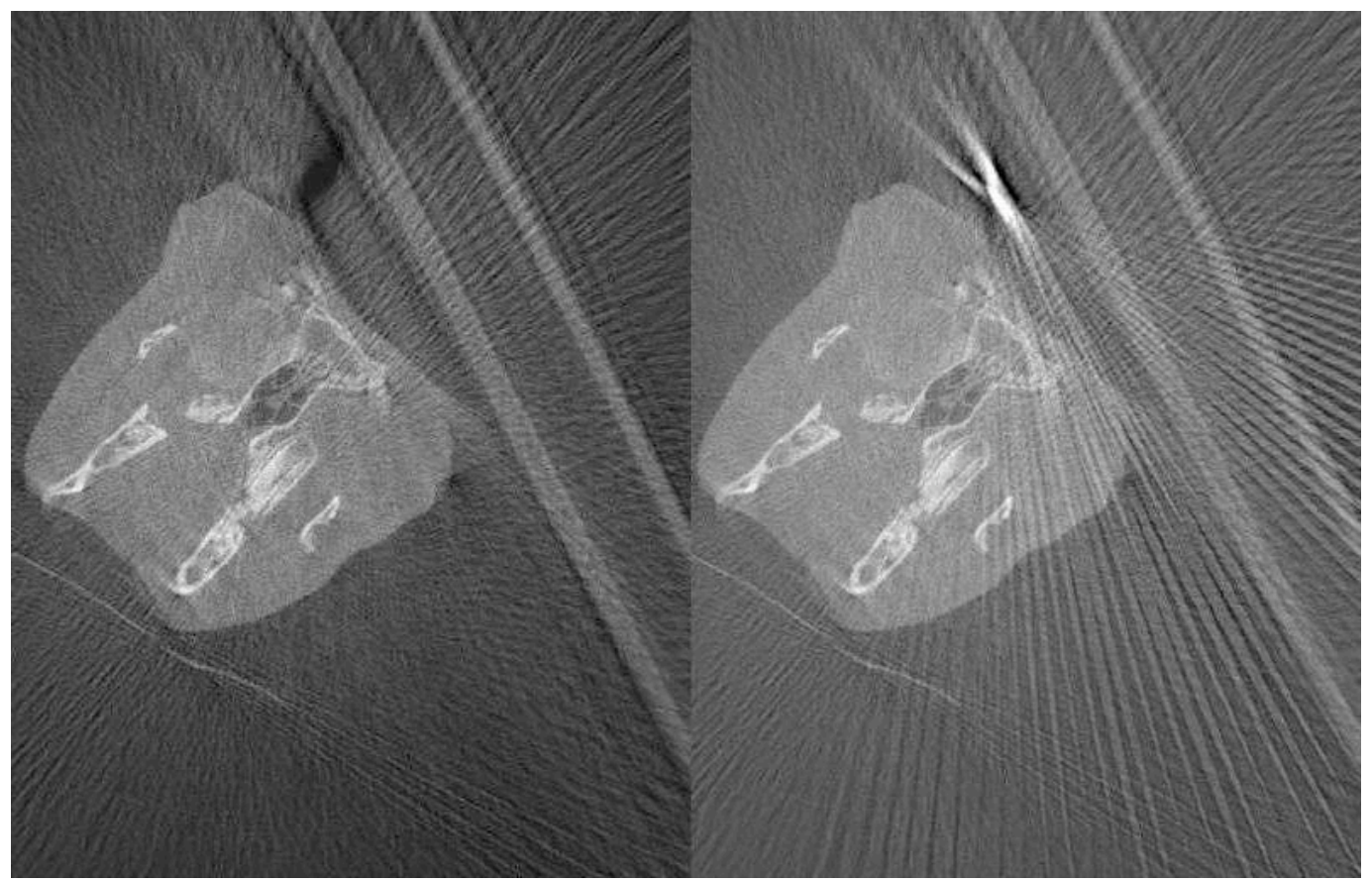

Figure 6. The resulting reconstruction with correction (left), and without correction (right). The lower row shows the enlarged regions of interest.

\section{DISCUSSION}

In this study, we have presented an efficient algorithm to reduce artifacts caused by high contrast objects. This novel approach identifies high contrast object in limited view reconstructions and then identifies the rays passing through the object using a computationally optimal approach based on a topological-walk technique coupled 
with use of a dual-space approach. These steps allow identification of the rays in effectively one step. Thus, no additional time is required in the reconstruction, e.g., as would be needed were the pixels corresponding to the high contrast identified using previously proposed techniques (see section 1).

Previous techniques rely on identification of the pixels in the images corresponding to the high contrast objects. This identification must be performed for each projection, a computation-intense process. In our approach, we work in 3D, performing a preliminary reconstruction and identifying the objects, and use a dualspace approach coupled with topological walk/pealing to identify the rays passing through the object. The beauty of this technique is that the dual-space/topological walk/pealing allows this ray identification in one step. Specifically, our underlying geometric algorithms are provably optimal in both time and space complexities and highly efficient. Therefore, our technique gives the possibility to remove different objects in the volume in real time.

Our results indicate that high quality reconstructions can be obtained using our technique. Note, due to the optimal time complexities of topological walk/peeling, the time for larger data sets will not increase much. This optimal time and space complexity and relative simple implementation makes our approach more attractive compared to other existing techniques.

\section{ACKNOWLEDGEMENTS}

This work was partly supported by The State University of New York at Buffalo - Interdisciplinary Research Development Fund, NSF grant IIS-0713489, NSF CAREER Award CCF-0546509, and the Toshiba Medical Systems Corporation.

\section{REFERENCES}

[1] Zhao, S., Robeltson, D., Wang, G., Whiting, B., and Bae, K., "X-ray ct metal artifact reduction using wavelets: an application for imaging total hip prostheses," Medical Imaging, IEEE Transactions on 19(12), 1238-1247 (Dec 2000).

[2] De Man, B., Nuyts, J., Dupont, P., Marchal, G., and Suetens, P., "Reduction of metal streak artifacts in x-ray computed tomography using a transmission maximum a posteriori algorithm," Nuclear Science, IEEE

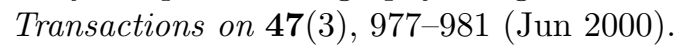

[3] Bal, M. and Spies, L., "Metal artifact reduction in ct using tissue-class modeling and adaptive prefiltering," Medical Physics 33(8), 2852-2859 (2006).

[4] Hsieh, J., Molthen, R. C., Dawson, C. A., and Johnson, R. H., "An iterative approach to the beam hardening correction in cone beam ct," Medical Physics 27(1), 23-29 (2000).

[5] Feldkamp, L. A., "Practical cone-beam algorithm," Journal of the Optical Society of America A, Optics and image science 1(6), 612-619 (1984).

[6] Noël, P., Corso, J., Xu, J., Hoffmann, K. R., Schafer, S., and Walczak, A., "Reconstruction from a flexible number of projections in cone-beam computed tomography via active shape models," In Press Medical Imaging 2009: Image Processing 7259, SPIE (2009).

[7] Chen, D. Z., Daescu, O., Hu, X. S., Wu, X., and Xu, J., "Determining an optimal penetration among weighted regions in two and three dimensions," in [SCG '99: Proceedings of the fifteenth annual symposium on Computational geometry], 322-331, ACM, New York, NY, USA (1999).

[8] Asano, T., Guibas, L. J., and Tokuyama, T., "Walking on an arrangement topologically," in [SCG '91: Proceedings of the seventh annual symposium on Computational geometry], 297-306, ACM, New York, NY, USA (1991). 